\title{
A cost effective topology migration path towards fibre
}

\author{
Frank Phillipson \\ TNO, Delft, the Netherlands \\ Email: frank.phillipson@tno.nl
}

\begin{abstract}
If an operator has as starting position a Full Copper topology in which ADSL or VDSL is offered from the Central Office, the next choice he has to make is to provide Full FttH or use an other topology option, e.g. FttCab, first as intermediate step to provide a next generation service package. In this paper we present a gradual topology migration path from Full Copper via FttCab and Hybrid FttH towards Full FttH. We look at the planning issues of this topology migration and the financial impact in comparison to the direct FttH roll-out. For this we present a case study in which we compare the costs of the presented gradual topology migration path to the alternative Full FttH direct option.
\end{abstract}

Index Terms-FttH, G.Fast, Telecommunications, access network planning, techno-economics.

\section{INTRODUCTION}

Internet at home is becoming as common as all other utility services. Every day more parties provide services on the Internet, but just as important for the bandwidth usage is the fact that those services are asking more bandwidth due to the integration of video into numerous services. On fixed connections we see that the bandwidth demand grows approximately $30 \%$ to $40 \%$ per year between now and 2020 . The current home connections of telecom operators are not prepared to offer this. The operators have to make the costly step to Fibre to the Cabinet (FttCab) or, even more costly, the step to Full Fibre to the Home (FttH). The roll out of Full FttH will be taking too long to compete with the cable TV operators, who can offer the required bandwidth at this moment.

In this paper we distinguish four topology types (see Fig. 1):

1) Full Copper: services are offered from the Central Office (CO) over a copper cable, using ADSL or VDSL techniques.

2) Fibre to the Cabinet (FttCab): the fibre connection is extended to the cabinet. From the cabinet the services are offered over the copper cable, using VDSL or G.Fast techniques.

3) Hybrid Fibre to the Home (Hybrid FttH): services are offered from a Hybrid FttH Node, which is connected by fibre, close to the customer premises, in the street or in the building.

4) Full Fibre to the Home (Full FttH): the fibre connection is brought up to the customer premises.

If an operator has as starting position the Full Copper topology in a certain area, he has to decide on the next step: bring the fibre connection all the way to the customers or

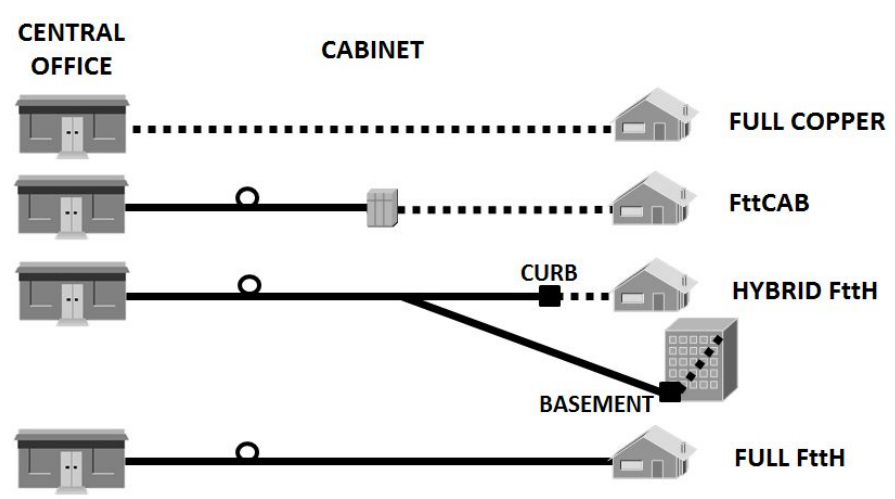

Fig. 1. Four topologies

use an intermediate step, where he brings the fibre closer to the customer, e.g. FttCab. To make this decision he has to look at the pros and cons of all the options. For example, the implementation of FttCab can be much faster than Full FttH, as it requires less digging, the last part of the connection from the street to the access node in the house does not have to be installed, and it meets the growing bandwidth demand for now and the near future. If, in future, this demand exceeds the supplied bandwidth, the remaining part to the residence can be connected with Full Fibre or using Hybrid Fibre as extra intermediate step. If the demand does not exceed the supplied bandwidth, for example it reaches some level of saturation, no further migration is needed, saving a lot of investments. However, when Full FttH is the expected final solution, using intermediate steps would incur investment and installation costs that might be lost and not reused. The copper technology that is required for the Hybrid FttH solution with the required bandwidth is currently developed and is named G.Fast. Results of this development make it plausible that Hybrid FttH using G.Fast is technically feasible up to $1 \mathrm{~Gb} / \mathrm{s}$ upto a copper distance of 200 meter. For this work and the technical issues look at the website of the CELTIC/4GBB project [1]. In this paper we present a gradual topology migration path from Full Copper to Full FttH, where we look at the planning issues of this migration and the financial impact. In this presented path we want to reuse tubes, cables and fibres or prepare them as much as possible. Preparing means that it is possible, 
for example, to put extra tubes in the ground when rolling out FttCab, that you will need in the case the full $\mathrm{FttH}$ step is made. This saves an extra digging activity later on. Of course not all equipment can be reused and not all preinvestments will be economical, but we will show that the postponement of huge investments will recover a part (or all) of these extra costs. First we start with a literature survey on techno-economical models. Next we describe the steps that have to be made in the presented gradual topology migration and what these steps mean for the planning process. The migration path we suggest is from Full Copper via FttCab and Hybrid FttH towards Full FttH. After that we will go into detail by presenting a cost model and show the results of a case study in which we compare the costs of the presented gradual topology migration path to the alternative Full FttH direct option.

\section{LITERATURE}

We look at the literature concerning techno-economics. In these papers the economic sanity of some choices are investigated. We are looking here at the techno economics of a migration path from Full Copper to Full FttH, using FttCap and Hybrid FttH as intermediate steps. The copper technology for Hybrid FttH, G.Fast, is not available yet in these kind of evaluations in the literature, although the migration or choices to be made in the other scenarios have been studied by many projects. The European projects ISTTONIC [2] and CELTIC-ECOSYS [3] resulted in various upgrade or deployment scenarios for both fixed and wireless telecommunication networks, published in [4] and [5]. A major question in these studies is when to make the decision to roll out a FttC/VDSL network or a Full FttH network. Based on demand forecasts, it was shown that it is profitable to start in dense urban areas, wait for five years and then decide to expand it to the urban areas. With the use of real option valuation the effect of waiting is rewarded to identify the optimal decision over time.

In [6] the OASE approaches are presented for more in depth analysis of the FttH total cost of ownership (TCO) and for comparing different possible business models both qualitatively and quantitatively.

The work of Casier [7] presents the techno-economic aspects of a fibre to the home network deployment. First he looks at all aspects of a semi-urban roll-out in term of dimensioning and cost estimation models. Next, the effects of competition are introduced into the analysis. Ref. [8] presents a multi-criteria model aimed at studying the evolution scenarios to deploy new supporting technologies in the access network to deliver broadband services to individuals and small enterprises. This model is based on a state transition diagram, whose nodes characterise a subscriber line in terms of service offerings and supporting technologies. This model was extended for studying the evolution towards broadband services and create the optimal path for broadband network migration. A same kind of model is presented in [9], where also an optimal strategy is proposed using a dynamic migration model. In all those papers G.Fast is not taken yet into consideration. Next to this, we think that incumbent telecom operators need all the effort to keep in track of the cable operators. There is almost no time for sophisticated strategies; they have to connect as much as possible of their clients with a sufficient high bandwidth connection.

\section{MigRATION STEPS}

In this paper we present a gradual topology migration path from Full Copper to Full FttH. In this section we will look at each step in this migration path and at the planning and dimensioning issues that play a role at each step. The four topologies under investigation are shown in Fig. 1. This leads to three topology migration steps that we discuss in this section:

1) From Full Copper to FttCab

2) From FttCab to Hybrid FttH

3) From Hybrid FttH to Full FttH

\section{A. Full Copper to FttCab}

When migrating from Full Copper to FttCab, it is necessary to extend the fibre further into the direction of the houses. Here the cabinet is selected as the next logical active point. Connecting the cabinet with fibre and installing the necessary hardware into it will be referred to as activating a cabinet from this point onwards. Doing this migration an operator does not want to activate all cabinets but only a selection. The operator wants to reach as many customers with as little investment as possible; usually the choice is made for a minimal penetration of, for example, $85 \%$. The operator will therefore look for a minimal cost selection of activated cabinets, that collectively have more than $85 \%$ of the customers within $1 \mathrm{~km}$, when the operator uses VDSL as technique. These cabinets are connected to the Central Office via new fibre optic links or circuits. These fibre optic circuits could have a maximum capacity in the number of cabinets that can be connected. The cabinets that are not activated will be connected to an activated cabinet using existing, or partly new, copper connections and are called 'placed in cascade'. Still, customers connected to these cabinets can be within $1 \mathrm{~km}$ from the activated cabinet and hence use VDSL.

If the operator wants to migrate to $\mathrm{FttCab}$, he has to design and plan the new network, starting with the available equipment and cables from the existing network, Full Copper, as shown in Fig. 2 and going towards FttCab, as shown in Fig. 3, where the use of rings is assumed. The operator has many design options to make. Methods for optimally connecting the cabinets can be found in literature. Gollowitzer et al. [10] present the Two Level Network Design (TLND) problem for greenfield deployments and roll-out mixed strategies of FibreTo-The-Home and Fibre-To-The-Curb, i.e., some customers are served by copper cables, some by fiber optic lines. In an other article [11] Gollowitzer gives an overview of MIP models for connected facility location problems. Here also only tree structures and uncapacitated nodes are considered. Mateus et al. [12] describe the network design problem of locating a set of concentrators which serves a set of customers with known demands. The uncapacitated facility location 
model is applied to locate the concentrators. Then, for each concentrator they analyse a topological optimization of its sub network based on a simple heuristic. In a third phase, they solve the upper level sub network connecting the concentrators to a root node in a tree structure. In [13] the topology design of hierarchical hybrid fibre-VDSL access networks is presented by Zhao et al. as an NP-hard problem. A complete strategy is proposed to find a cost-effective and high-reliable network with heuristic algorithms in a short time. The Ant Colony Optimization (ACO) has been implemented for a clustering problem. The network structure they look at is a two layer street cabinet solution with Branch Micro Switches (BMS) and Lead Micro Switches (LMS) where the BMS is connected with the CO with two paths and the LMS is connected to two BMSs. The users are connected in a star with one LMS. The major planning problem here is to build up the intermediate BMS level. A last example is the paper of Baldacci et al. [14] where they present the Capacitated m-Ring-Star Problem to connect a set of customers partly by a ring structure and partly by a tree structure.

Our approach is presented in (in press) [15] and (unpublished) [16]. Assuming a minimum penetration of customers within $1 \mathrm{~km}$ and the need for rings that have all disjoint paths, we found this to be too complex to solve in one step. Therefore we divided this complex problem into three simpler sub-problems, which are already NP-hard. These three subproblems in our approach are:

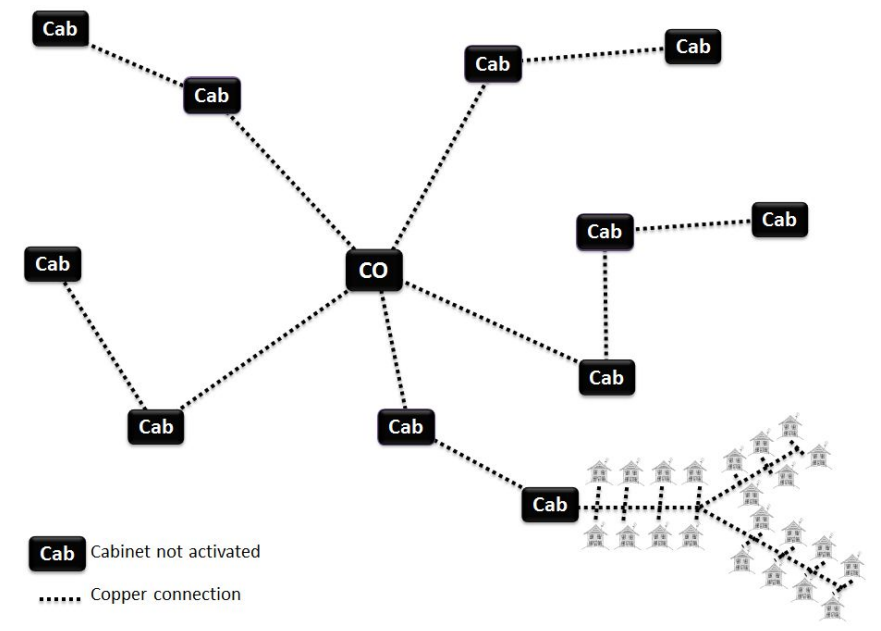

Fig. 2. Starting point: Full Copper

1) Which cabinets must be activated in order to reach the desired percentage of households at minimal costs? Fig. 2 shows the starting point. All cabinets are connected through copper with the Central Office (CO). Several residences are connected to the cabinet; this is only shown for one cabinet in the illustration. Now a subset of the cabinets needs to be activated in order to reach the intended number of households over copper from an activated cabinet within the set distance.

2) Which cabinet is served by which fibre optic circuit? The cabinets now have to be divided into groups in order to determine which cabinets will be jointly connected by one fibre optic circuit.

3) How will each fibre circuit run? The physical route of the fibre optic circuits needs to determined. What order will they be connected in, and how does this route run? How to make sure that no track is used twice in one circuit, see Fig. 3?

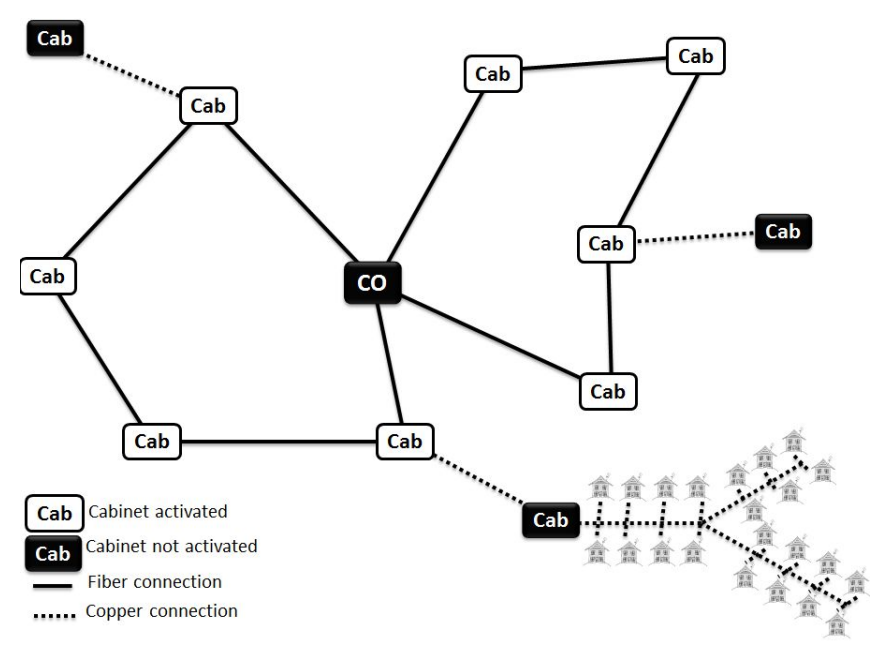

Fig. 3. Physical route of the fibre optic circuit in FttCab.

\section{B. FttCab to Hybrid FttH}

When we look in more detail to this next part of the copper network we see a situation as shown in Fig. 4. This is a typical situation in the last mile of the Dutch copper network: a heavily branched network. In this network new network nodes have to be placed for the G.Fast technology. To do this, possible locations for these network nodes have to be determined, logical places are the dots in the figure, the branching points of the network. It is known which houses are connected to these locations at which distance. Now one should decide which locations will be used and how they are connected to a fibre node.

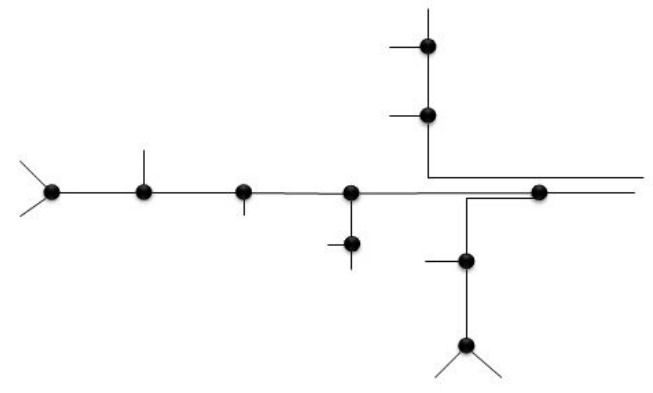

Fig. 4. Typical last mile in the Netherlands

Some choices can be made in this process:

1) Should all houses be reached from a Hybrid Fibre node within a fixed distance, or a fixed percentage of houses, 
or do we have a fine for every house not connected within that certain distance? We distinguish:

a) All houses must be connected, a fine is considered otherwise.

b) A certain percentage has to be within the defined distance.

2) Does the node have a capacity restriction?

a) Yes

b) No

3) How are the nodes connected:

a) Tree or star structure

b) Ring structure

In this paper we assume all houses have to be connected, we assume there is a capacity restriction at the Hybrid FttH node and that the Hybrid FttH nodes are connected by a tree structure. For the full analysis of all eight scenario's and how to plan this, look at [17]. Note that it might be possible that the current cabinet location is also a possible Hybrid FttH node location. This is when all of the connections in at least one of the bundles going out of the cabinet are within the fixed distance.

\section{Hybrid FttH to Full FttH}

At this stage the last 20-200 meters have to be provided with fibres. Next to this, in the case a Point-to-Point (PtP) fibre connection is preferred, per connection a fibre has to be provided from the, new, fibre PoP to the Hybrid FttH node, where a splice can be made to the fibre cable for the last 200 meters. The needed ducts can be placed earlier, as we discuss in the next section.

\section{Dimensioning Looking Ahead}

If an operator follows the the gradual topology migration path he better takes the possible next steps into account when planning the first step, FttCab. To do this he can take the following steps:

Step 1: Dimensioning the circuit. When the operator makes the step to FttCab, the maximum number of customers per $\mathrm{FttCab}$ circuit, say $K$, is related to the number of customers connected to a Full FttH-PoP, call that $X$. This Full FttH-PoP (PoP in the remaining of the paper) will be necessary in the case Full FttH is rolled out and the cabinets are not big enough to handle the active equipment. The parameters $X$ and $K$ together determine the number of PoPs per circuit:

\begin{tabular}{|l|l|l|l|}
\hline & clients/PoP & clients/ring & PoPs/ring \\
\hline 1 & $0<X<0.5 K$ & $\lfloor K / X\rfloor * X$ & $\lfloor K / X\rfloor$ \\
\hline 2 & $0.5 K<X<K$ & $X$ & 1 \\
\hline 3 & $X>K$ & $K$ & $<1$ \\
\hline
\end{tabular}

Suppose $X=500$ and $K=2900$ : Here situation 1 is applicable: there will be $\lfloor 2900 / 500\rfloor * 500=2500$ customers allowed on the circuit and five PoPs. See Fig. 5.

Suppose $X=1500$ and $K=2900$ : Here situation 2 is applicable: there will be 1500 customers allowed on the circuit and one PoP.

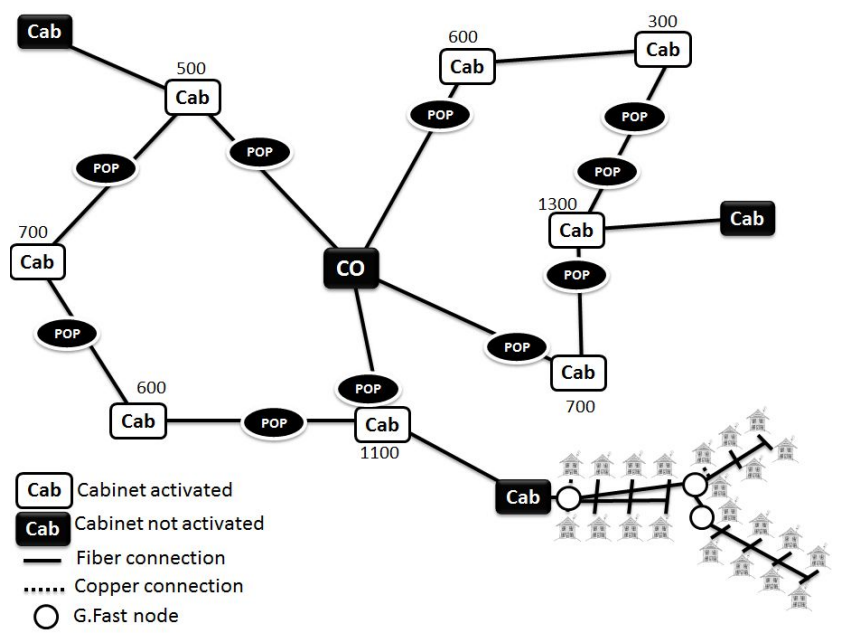

Fig. 5. Example with $X=500, K=2900$

Suppose $X=6000$ and $K=2900$ : There will be 2900 customers admitted to the circuit. The CO location will (if possible) service numerous circuits and (possibly) contain numerous PoPs. See Fig. 6.

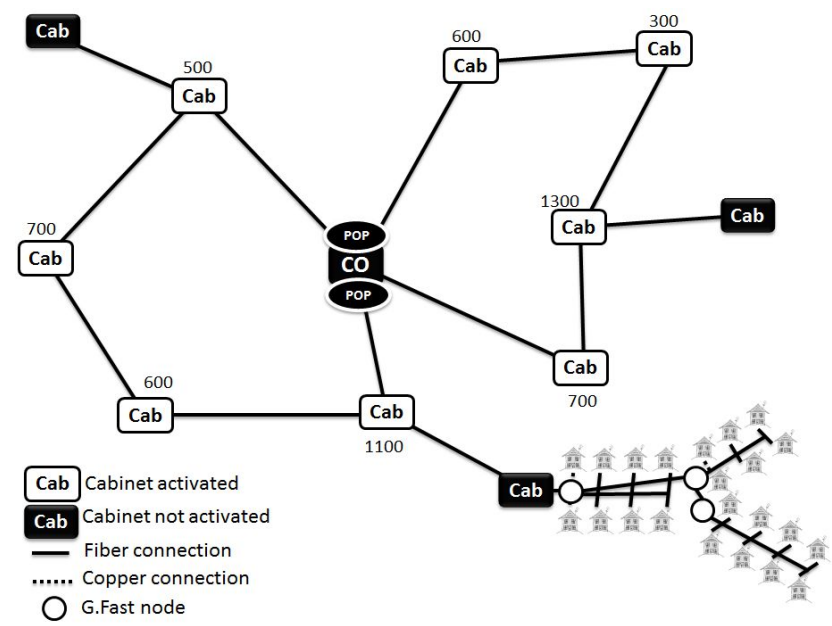

Fig. 6. Example with $X=6000, K=2900$

Step 2: Setting up FttCab architecture. The architecture of the FttCab implementation can now be determined using the method described in [16]. When clustering the cabinets, a precondition should be the number of customers (maximum) on the fibre circuit, and therefore the cluster, of step 1. When creating the circuit it should be taken into consideration that the circuit will go through the cabinets and through the determined number of Full FttH POP location(s) from step 1. Here also extra ducts should be placed for latter Full (PtP) FttH delivery.

Step 3: Setting up Hybrid FttH architecture. Here the duct structure has to be continued to the Hybrid FttH node. Not only the (possible) one fibre is needed to connect the Hybrid FttH node, but be prepared for the Full (PtP) Fibre roll-out. 
Step 4: Transition to Full FttH. When the time has come to transmigrate to Full $\mathrm{FttH}$, two situations might be possible:

1) More than one Full FttH-PoP per ring: Every residence receives the fibre optic connection to the original cabinet. Households (or cabinets) are allocated to Full FttH POP in such a manner that the total distance is minimized (within capacity limitations).

2) One or less Full FttH-PoP per circuit: Households receive fibre optic connection according to original $\mathrm{CO}$ location.

\section{COST MODEL}

In this section we present a cost model to compare two topology migration paths with regard to costs. This cost model is part of the techno-economic model that was built in the CELTIC/4GBB project. The model is described in [18]. More results from the cost model or the total techno-economic analysis can be found in [19].

\section{A. Assumptions}

For the calculations of the cost of each topology migration path we assume a certain topology roll out, with choices regarding technology, structure, dimensioning etcetera. We assume that a G.Fast node has a capacity of $X_{3}$ connections, that $X_{2}$ G.Fast nodes can be connected to one cabinet and that $X_{1}$ cabinets can be connected to one ring to the central office. This makes the total connections on a cabinet $X_{2}$ times $X_{3}$. This is shown in Fig. 7. For example, a FttCab fibre ring has a maximum of 2500 connections, divided in 6 cabinets, each with capacity of 384 connections, connected by a fibre ring. When the migration from FttC to Hybrid FttH is performed, each cabinet gets 8 Hybrid FttH nodes, each having 48 connections, connected in a star structure. The fibre ring can be fed by one FttH PoP, the location of this PoP is already determined and taken into the ring. This is shown in Fig. 7.

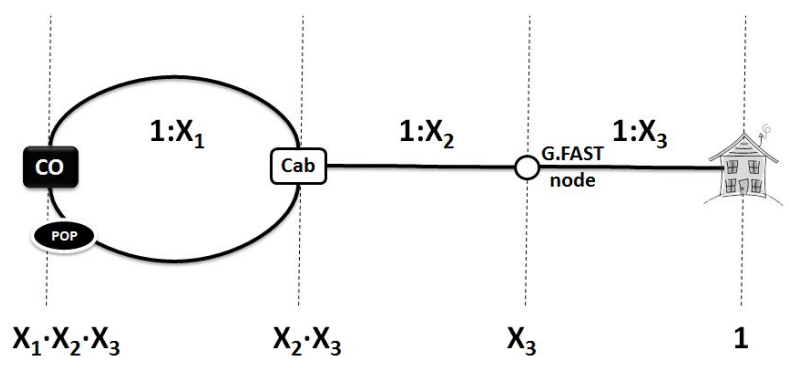

Fig. 7. Design choices of the network

\section{B. Geometric model}

For the cost model we need to calculate distances of both trench and cable. Next to that, we like to know something about the (expected) maximum distance in the roll out. For these calculations we use a geometric model, as also used in e.g. [7]. We distinguish four main variants in this geometric model:

1) Star structure, single sided houses.

2) Star structure, double sided houses.

3) Snake structure, single sided houses.

4) Snake structure, double sided houses.
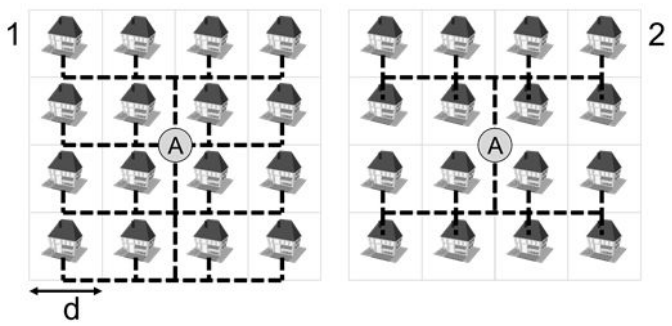

3

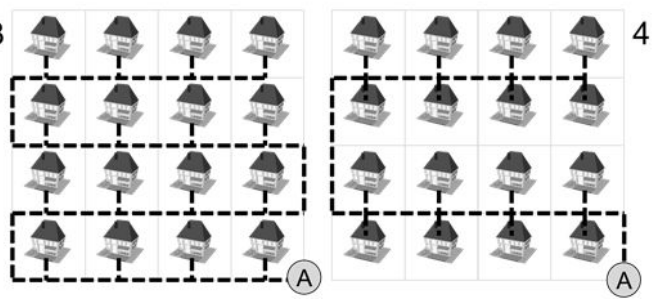

Fig. 8. Four geometric models

These four structures are shown in Fig. 8. We define $A$ as the access point, $n$ as the number of houses to be connected, $s=\sqrt{n}$ and $d=$ width of the premises. For each of these structures the length of the trench and cable (without gardens) can be calculated:

1) Star structure, single sided houses: distance of digging = $d *(s+1)(s-1)$, distance of cable $=2 * d * s *\left\lceil\frac{1}{2} s\right\rceil *\left\lfloor\frac{1}{2} s\right\rfloor$.

2) Star structure, double sided houses: distance of digging $=d *\left(\frac{1}{2} s *(s-1)+s-2\right)$, distance of cable $=2 * d *$ $s *\left\lceil\frac{1}{2} s\right\rceil *\left\lfloor\frac{1}{2} s\right\rfloor$.

3) Snake structure, single sided houses: distance of digging $=d *\left(\left(s^{2}-\frac{1}{2}\right)+(s-1)\right)$, distance of cable $=d *\left(\frac{1}{2} s^{3}+\right.$ $\left.\frac{1}{2} s^{2}\left(s^{2}-1\right)\right)$.

4) Snake structure, double sided houses: distance of digging $=d *\left(\left(\left\lceil\frac{1}{2} s\right\rceil * s-\frac{1}{2}\right)+(s-1)\right)$, distance of cable $=$ $d *\left(s^{2}+\frac{1}{2} s^{3}+K * s *(s+2)\right) \approx d * \frac{1}{4}(s+2) * s^{3}$, where $K=\frac{1}{2}(\lceil s / 2\rceil) *(\lceil s / 2\rceil-1)+\frac{1}{2}(\lfloor s / 2\rfloor) *(\lfloor s / 2\rfloor-1)$

The maximum copper distance can be calculated by:

1) Star structure, single sided houses: $s * d-0.5 d$.

2) Star structure, double sided houses: $2\left\lceil\frac{1}{2} s-1\right\rceil * d+0.5 d$.

3) Snake structure, single sided houses $s^{2} * d+s * d-1.5 d$.

4) Snake structure, double sided houses $\left\lceil\frac{1}{2} s\right\rceil * s * d+2 *$ $\left\lceil\frac{1}{2} s\right\rceil * d-1.5 d$.

For a multi layer network, each layer can be treated as a separate geometric model. In the architecture as shown earlier three layers can be distinguished:

1) The $\mathrm{CO}$ as the access node and the 8 cabinets to be connected.

2) The cabinet as access point and the 8 Hybrid FttH nodes to be connected. 
3) The Hybrid FttH node as the access node and the 48 houses to be connected.

In this analysis we assume for layer 1 a ring structure, for layer 2 a star structure, single side, and for layer 3 a snake structure double sided, as seen in some European countries like The Netherlands.

\section{Cost Parameters}

We distinguish the following cost categories:

1) Connection $\mathrm{CO}$ to cabinet: Digging, closing trench, breaking and repairing tiles; ducts.

2) Equipment and (de-) installation cabinet

3) Connection cabinet to Hybrid FttH node: Digging, closing trench, breaking and repairing tiles; ducts.

4) Equipment and (de-) installation Hybrid FttH node

5) Connection Hybrid FttH node to premises: Digging, closing trench, breaking and repairing tiles; direct buried cable.

6) Equipment and (de-) installation in premises

The used values are ${ }^{1}$ :

\begin{tabular}{|l|r|l|}
\hline Description & costs & unit \\
\hline Digging, closing trench & 15 & $€ / \mathrm{m}$ \\
\hline Breaking and repairing tiles & 10 & $€ / \mathrm{m}$ \\
\hline Fibre (Direct buried cable) & 0.3 & $€ / \mathrm{m}$ \\
\hline Drilling (garden) & 25 & $€ / \mathrm{m}$ \\
\hline Duct & 2 & $€ / \mathrm{m}$ \\
\hline Blowing fibre or cable & 500 & $€ /$ duct \\
\hline Hybrid FttH node E \& I & 2500 & $€ /$ node \\
\hline Cabinet E \& I & 11000 & $€ /$ node \\
\hline Premises E \& I & 250 & $€ /$ node \\
\hline Removing equipment & 250 & $€ /$ node \\
\hline End user equipment & 100 & $€ /$ conn. \\
\hline
\end{tabular}

\section{Validation}

For a rough validation we look at the results of earlier work (unpublished) [17]. Here we calculated the cost for two cities in the Netherlands, Amsterdam and The Hague, in detail, using the activation algorithm [16] and Prim's algorithm [20]. We assume that the cabinets already have a fibre connection, so our focus is the part of the network between the cabinet and the home connection. The Amsterdam case has 150,058 branching point, The Hague 89,076. Those branching points are the potential spots to place the new equipment. In both cities we want to connect at least $99 \%$ of the customers within 200 meter to a G.Fast node. Each G.Fast node is placed in a manhole. We can place each combination of 16-port and 48port G.Fast equipment (G.Fast multiplexer) in the manhole. Now we plot the resulting costs per FttCab connection of the various central offices and their average connection density in Fig. 9 and compared it with the results of the simple geometric model. For both cities we plot a logarithmic trend line to indicate the underlying relationship. The differences between Amsterdam and The Hague follow from the size of

\footnotetext{
${ }^{1}$ These values come from the TNO cost database, filled by input of various constructors
}

the cabinets. In The Hague the current cabinet size is bigger; this increases the average length between the cabinet and the new activated points.

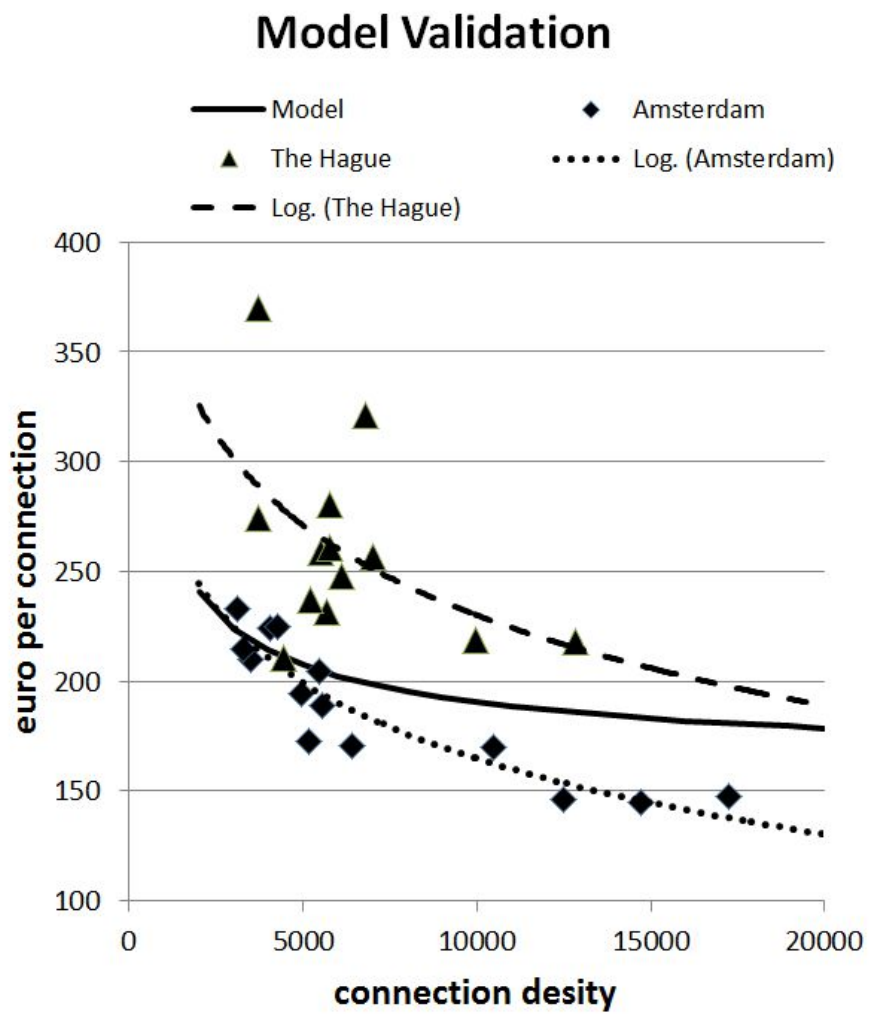

Fig. 9. Validation of the model

\section{E. Results}

We use the cost parameters as presented in the previous section and the distances calculated using the models and assumptions presented earlier. The area we look at has a density of 6000 connections per square kilometre, a city centre. These assumptions are representative for the Dutch case, but also for other countries where the last 20-200 meters are constructed by underground cables. We take an area with 2304 connections, which is 1 full $\mathrm{CO}$ and a G.Fast node capacity of 48 connections.

The first topology migration path under consideration is the presented gradual topology migration path, from Full Copper (FC) to Full FttH (FF), using FttCab (FCab) and Hybrid FttH (HF) as intermediate steps. In the next table, the costs of the three steps in the topology migration path are shown. The categories are those of the previous section: 


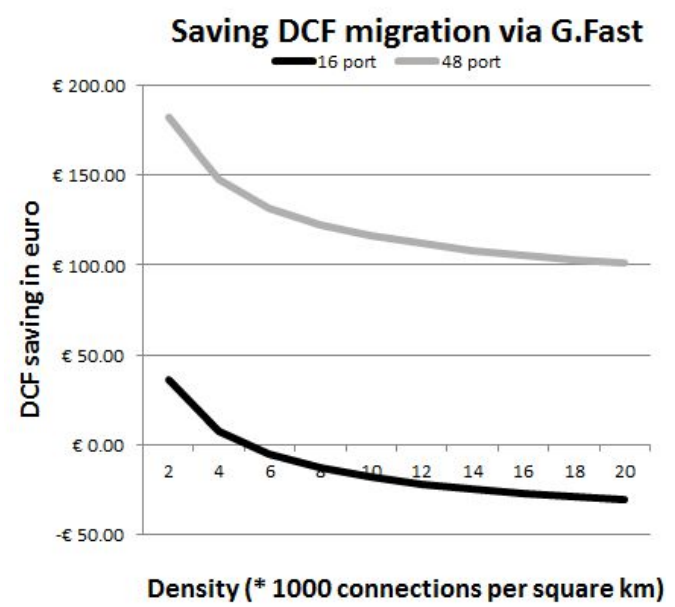

Fig. 10. Saving DCF migration path

\begin{tabular}{|l|r|r|r|}
\hline Category & FC to FCab & FCab to HF & HF to FF \\
\hline$(1)$ & $€ 87,930$ & $€ 3,000$ & \\
\hline$(2)$ & $€ 60,000$ & $€ 1,500$ & \\
\hline$(3)$ & & $€ 98,545$ & $€ 24,000$ \\
\hline$(4)$ & & $€ 76,800$ & $€ 12,000$ \\
\hline$(5)$ & & & $€ 1,434,359$ \\
\hline$(6)$ & $€ 115,200$ & $€ 230,400$ & $€ 806,400$ \\
\hline Total & $€ 263,130$ & $€ 410,245$ & $€ 2,276,759$ \\
\hline Per connection & $€ 114$ & $€ 178$ & $€ 988$ \\
\hline
\end{tabular}

This includes $2 \%$ inflation and adds up to a total of $€ 1,280$ per connection when the total topology migration path is followed. Note that bringing only FttCab and Hybrid FttH to the customers is relatively cheap, only $€ 292$ for bringing already a high bandwidth. The roll-out of Full FttH directly migration will lead to the following calculated costs:

\begin{tabular}{|l|r|}
\hline Category & Full FttH \\
\hline CO to premises & $€ 1,424,229$ \\
\hline Premises & $€ 806,400$ \\
\hline Total & $€ 2,230,629$ \\
\hline Per connection & $€ 968$ \\
\hline
\end{tabular}

This is a total cost of $€ 968$ per connection. Around $30 \%$ cheaper. But now will use the discounted cash flow (DCF) method to compare the two outcomes. The used weighted cost of capital (WACC) for fixed telecom operators comes from [21] and is 7.38\%. If we assume that the investment for FttCab has to be made next year, the migration to Hybrid FttH will be in five years (on average) and the migration to FttH will be in 10 years (on average) and compare this to a Full FttH roll out next year (again on average) the cost comparison is totally different, regarding to the discounted cash flow (DCF):

\begin{tabular}{|l|r|r|r|r|r|}
\hline Migration & $\begin{array}{r}\text { FC to } \\
\text { FCab }\end{array}$ & $\begin{array}{r}\text { FCab } \\
\text { to HF }\end{array}$ & $\begin{array}{r}\text { Hf to } \\
\text { FF }\end{array}$ & $\begin{array}{r}\text { FC to } \\
\text { FF }\end{array}$ & Total \\
\hline Gradual & $€ 114$ & $€ 137$ & $€ 585$ & & $€ 836$ \\
\hline Full FttH & & & & $€ 968$ & $€ 968$ \\
\hline
\end{tabular}

Now the migration path is cheaper, $€ 836$ against $€ 968$ in net present value.
In Fig. 10 we see the difference in discounted costs for different values of density of connection, ceteris paribus, and in Fig. 11 the maximum copper length with different density of connections, ceteris paribus. Both are calculated for both a 16-port G.Fast node and a 48-port G.Fast node. This are the two options that are under consideration of the manufacturing parties. We can conclude that, with the chosen parameters the migration path is cheaper in DCF than the direct roll-out of Full FttH for the 48-port. In the case of the 16-port the break-even point is touched at 6000 connections per square kilometre.

Some observations:

- With a star structure the distances will be shorter so this can serve more nodes. The Dutch situation (see Fig. 4) is heavily branched which has both characteristics of snake and star networks. You can find points in this network from where it looks like a star structure further on in the direction of the customer.

- Bonding, combining multiple wire pairs to increase available bandwidth, will reduce the capacity (in connections) of the node.

- The maximum copper distance used for VDSL varies between countries and studies. In the Netherlands a maximum of 1000 meters is used, whereas in practice in the cities a large part is within 200 meters. The study presented in [13], as discussed earlier, adopt a network structure with a two layer cabinet solution with Branch Micro Switches (BMS) and Lead Micro Switches (LMS) where the BMS is connected with the CO with two paths and the LMS is connected to two BMSs. The users are connected in a star with one LMS with a typical distance of 100-300 meter. [4] see a typical maximum VDSL distance of 400 meter. This all indicates that in several countries a roll-out with Hybrid FttH nodes at the current cabinet is possible in a big part of the cases.

- We compared the two topology migration paths until Full FttH. However, the gradual topology migration path has the option that on of the intermediate steps will be the final solution if a level of saturation in bandwidth demand is reached. This leads to lower expected costs or some real option value (see for example [22]), that was not taken into account in our approach. This could justify the 16-port case. Next to this, a fast roll-out of FttCab could save the market share of the operator, see for example [23].

- From technical point of view, the 48-port modem is not just a combination of 3 16-port modem. To serve a bigger group connections that share a cable, they should be served from the same modem that makes complex calculations to reduce the crosstalk effects.

- A point of concern is that in case of a 48-port G.Fast modem not all connections are within 200 meters over copper from the Hybrid FttH node (see Fig. 11), which is, about, a bound for the high bandwidth using G.Fast, see [24]. In a case study of Amsterdam [17], based on real distances, locations of cabinets and copper cables we 


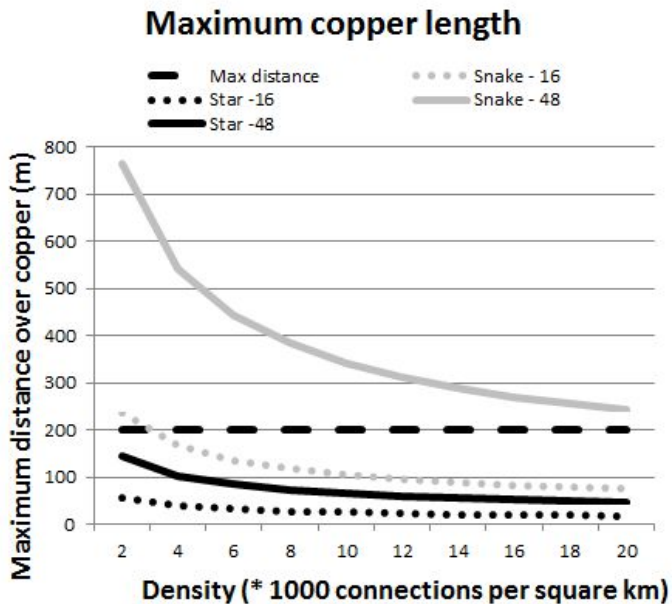

Fig. 11. Maximum copper length snake structure

saw an average utilisation of the G.Fast node of 38 ports to serve $99 \%$ of the connections within 200 metres.

\section{SUMmARY AND CONCLUSIONS}

We looked at the economics and planning issues of a full migration path from ADSL to FttH, using FttC and Hybrid $\mathrm{FttH}$ as intermediate steps. We outlined a possible migration path that can be used in practice. We discussed some planning issues that arise in each migration step and the precautionary measures that have to be taken for steps in the future. We presented a cost model to calculate the differences between the proposed migration path and the one-step Full FttH rollout and showed that, under our assumptions, which are representative for the Dutch case, but also for other countries where the last 20-200 meters are constructed by underground cables, the migration path is economically feasible. For an operator this is important information: bringing Hybrid $\mathrm{FttH}$ is a relatively cheap option to deliver high bandwidths quickly. If, however, this solution will be insufficient in the future, the postponement of the investment of $\mathrm{FttH}$ gives a cost saving that is big enough to compensate for the extra costs of the (possible needed) full migration path.

\section{REFERENCES}

[1] CELTIC-4GBB. [Online]. Available: www.4gbb.eu

[2] IST-TONIC. [Online]. Available: www.ist-tonic.org

[3] CELTIC-ECOSYS. [Online]. Available: www.celtic-initiative.org/ projects/ecosys

[4] T. Rokkas, D. Katsianis, and D. Varoutas, "Techno-economic evaluation of fttc/vdsl and ftth roll-out scenarios: Discounted cash flows and real option valuation," J. OPT. COMMUN. NETW., vol. 2, no. 9, pp. 760771, 2010.

[5] T. Monath, N. K. Elnegaard, P. Cadro, D. Katsianis, and D. Varoutas, "Economics of fixed broadband access network strategies," IEEE COmmunications Magazine, pp. 132-139, 2003.

[6] S. Verbrugge, K. Casier, B. Lannoo, C. M. Machuca, T. Monath, M. Kind, and M. Forzati, "Research approach towards the profitability of future ftth business models," in Proceedings Telecommunications Network Strategy and Planning Symposium (NETWORKS), 2011.

[7] K. Casier, Techno-Economic Evaluation of a Next Generation Access Network Deployment in a Competitive Setting. Universiteit Gent, 2009.

[8] C. H. Antunes, J. F. Craveirinha, and J. N. Climaco, "Planning the evolution to broadband access networks: A multicriteria approach," European Journal of Operational Research, vol. 109, pp. 530-540, 1998.
[9] R. Zhao, L. Zhou, and C. M. Machuca, "Dynamic migration planning towards ftth," in Telecommunications Network Strategy and Planning Symposium (NETWORKS), 2010.

[10] S. Gollowitzer, L. Gouveia, and I. Ljubić, "A node splitting technique for two level network design problems with transition nodes," Lecture Notes in Computer Science, vol. 6701, pp. 57-70, 2011.

[11] S. Gollowitzer and I. Ljubić, "Mip models for connected facility location: A theoretical and computational study," Computers and Operations Research, vol. 38, pp. 435-449, 2011.

[12] G. R. Mateus, F. R. B. Cruz, and H. P. L. Luna, "An algorithm for hierarchical network design," Location Science, vol. 2, no. 3, pp. 149164, 1994.

[13] R. Zhao, H. Liu, and R. Lehnert, "Topology design of hierarchical hybrid fiber-vdsl access networks with aco," in Proceedings of Fourth Advanced International Conference on Telecommunications, 2008.

[14] R. Baldacci, M. Dell'Amico, and J. S. González, "The capacitated mring-star problem," Operations Research, vol. 55, no. 6, pp. 1147-1162, 2007.

[15] F. Phillipson, "Efficient clustering of cabinets at FttCab," in The 13th International Conference on Next Generation Wired/Wireless Advanced Networking (NEW2AN 2013), St.Petersburg, Russia, 2013.

[16] — , "Fast roll-out of fibre-to-the-cabinet: optimal activation of cabinets," In preparation, 2013.

[17] _ - "Planning of fibre to the curb using g.fast in multiple rollout scenarios," in 3rd International Conference on Information Communication and Management (ICICM2013), Paris, France, 2013.

[18] K. Ahmed, P. Verhagen, F. Phillipson, and C. Smit, "4gbb technoeconomic feasibility and regulatory issues,part 3 - techno economic modeling - tno approach," TNO - CELTIC/4GBB, Tech. Rep., 2012.

[19] R. van den Brink and F. Phillipson, "4gbb techno-economic feasibility and regulatory issues, part 4 - techno economic calculations - tno approach," TNO - CELTIC/4GBB, Tech. Rep., 2012.

[20] R. C. Prim, "Shortest connection networks and some generalizations," Bell Systems Technical Journal, vol. 36, p. 13891401, 1957.

[21] A. Mason, Conceptual approach for the fixed and mobile BULRIC models. OPTA, 2010.

[22] J. Alleman, "A new view of telecommunications economics," Telecommunications Policy, vol. 26, pp. 87-92, 2002.

[23] F. Phillipson, C. Smit-Rietveld, and P. Verhagen, "Fourth generation broadband delivered over copper - a techno-economic study -," In preparation, 2013.

[24] R. F. van den Brink, "Enabling 4gbb via the last copper drop of a hybrid $\mathrm{ftth}$ deployment," Broadband Journal of the SCTE, vol. 33, no. 2, pp. 40-46, 2011.

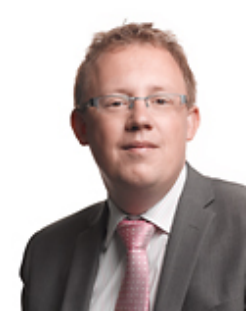

Frank Phillipson (1973), studied Econometrics at the Vrije Universiteit Amsterdam, and wrote his Master's thesis in the field of Operations Research in 1996. In the same year he joined the Delft University of Technology to follow the Post-Doctoral course Mathematical Design Engineering with a strong focus on application of Operations Research techniques in networks. From 1998 until 2003 he was employed at KPN Research. In 2002, KPN placed its research department in TNO, the largest applied research institute in the Netherlands, where Frank is currently working in the department 'Performance of Networks and Systems'. There he is particularly working in the field of planning of ICT/telecom and electricity networks. In addition to this main topic, he has worked on projects for financial and economic models relating to telecom business. This has provided him a good overview of the technical as well the economic impact of network planning and dimensioning. Frank Phillipson is co-author of several papers and has supervised many Masters students working on their thesis at TNO. 